\title{
Free and forced convective flow in a vertical channel filled with composite porous medium using Robin boundary conditions
}

\author{
Jada Prathap Kumar, Jawali Channabasappa Umavathi", Yadav Ramarao
}

Department of Mathematics, Gulbarga University, Karnataka, India

Email address:

jc_uma11@yahoo.com (J. C. Umavathi)

\section{To cite this article:}

Jada Prathap Kumar, Jawali Channabasappa Umavathi, Yadav Ramarao. Free and Forced Convective Flow in a Vertical Channel Filled with Composite Porous Medium Using Robin Boundary Conditions. American Journal of Applied Mathematics.

Vol. 2, No. 4, 2014, pp. 96-110. doi: 10.11648/j.ajam.20140204.11

\begin{abstract}
Mixed convection flow and heat transfer in a vertical channel filled with composite porous medium using Robin boundary conditions is analyzed. The flow is modeled using the Darcy-Lapwood-Brinkman model. The viscous and Darcy dissipation terms are included in energy equation. The plate exchanges heat with an external fluid. Both the conditions of equal and different reference temperature of the external fluid are considered. The governing equations are coupled and non-linear because of inclusion of dissipation terms and buoyancy forces. The equations are solved using perturbation method valid for small values of perturbation parameter. However, the restriction on the perturbation parameter is relaxed by finding the solutions of governing equations by using Differential Transform Method. The effects of various parameters such as mixed convection parameter, porous parameter, viscosity ratio, width ratio, conductivity ratio and the Biot numbers on the flow are discussed. The percentage of error between perturbation method and differential transformation method increases as the perturbation parameter increases for both equal and unequal Biot numbers.
\end{abstract}

Keywords: Mixed Convection, Composite Porous Medium, Perturbation Method, Differential Transform Method, Robin Boundary Condition

\section{Introduction}

Convective heat transfer in closed conduits partially filled with a porous medium is of essential importance to a variety of engineering applications including solar collectors, micro scale channels for cooling electronic components, nuclear reactors, chemical catalytic reactors, thermal insulation, and heat pipes. In the past decade, this importance has attracted substantial analytical studies. The flow and heat transfer aspects of immiscible fluids is of special importance in petroleum extraction and transport. For example, the reservoir rock of oil always contains several immiscible fluids in its pores. Part of the pore volume is occupied by water and the rest may be occupied either by oil or gas or both. These examples show the importance of knowledge of the laws governing immiscible multi-phase flows for proper understanding of the processes involved. The subject of two-fluid flow and heat transfer has been extensively studied due to its importance in chemical and nuclear industries. Identification of the two-fluid flow region and determination of the pressure drop, void fraction, quality reaction and two-fluid heat transfer coefficient are of great importance for the design of two-fluid systems. In modeling such problems, the presence of a second immiscible fluid phase adds a number of complexities as to the nature of interacting transport phenomena and interface conditions between the faces.

The work of Beavers and Joseph [1] was one of the first attempts to study the fluid flow boundary conditions at the interface region. They performed experiments and detected a slip in the velocity at the interface. Neale and Nadar [2] presented one of the earlier attempts regarding this type of boundary condition in porous medium. In this study, the authors proposed continuity in both the velocity and the velocity gradient at the interface by introducing the Brinkman term in the momentum equation for the porous side. Vafai and Kim [3] presented an exact solution for the fluid flow at the interface between a porous medium and a fluid layer including the inertia and boundary effects. In 
this study, the shear-stress in the fluid and the porous medium were taken to be equal at the interface region. Vafai and Thiyagaraja [4] analytically studied the fluid flow and heat transfer for three types of interfaces, namely, the interface between two different porous media, the interface separating a porous medium from a fluid region and the interface between a porous medium and an impermeable medium. Continuity of shear stress and heat flux were taken into account in their study while employing the Forchheimer-extended Darcy equation in their analysis. Other studies considered the same set of boundary conditions for the fluid flow and heat transfer used in Vafai and Thiyagaraja [4] such as Vafai and Kim [5], Kim and Choi [6], Poulikakos and Kazmierczak [7] and Ochoa and Whitaker [8].

Forced convection in composite channels is a subject of intensive investigation. This is due to rapid development of technology and numerous modern thermal applications relevant to this area, such as cooling of micro-electronic devices. Some novel designs of heat sinks for cooling micro-electronic devices utilize highly porous materials, such as aluminum foam [9]. Poulikakos and Kazmierczak [7] presented analytical solutions for forced convection flow in ducts where the central part is occupied by clear fluid and the peripheral part is occupied by a BrinkmanDarcy fluid saturated porous medium. The results of Poulikakos and Kazmierczak [7] were extended by Kuznetsov [10] to account for the Forchheimer (quadratic drag) effects. Nield and Kuznetsov [11]) considered a forced convection problem in a channel whose centre is occupied by a layer of isotropic porous medium and whose peripheral part is occupied by another layer of isotropic porous medium, each of the layers with its own permeability and thermal conductivity. They utilized the Darcy law for the flow in porous layers. Malashetty et al. $[12,13]$ studied mixed convection in an inclined channel containing porous medium for immiscible fluids. Umavathi et al. [14-16] studied the flow and heat transfer in a composite channel. Recently, Prathap Kumar et al. [17,18] studied fully developed mixed convection flow in a vertical channel for composite porous medium for symmetric and asymmetric wall heating conditions.

In the past, the laminar forced convection heat transfer in the thermal entrance region of a rectangular channel has been analyzed either for the temperature boundary condition of the first kind, characterized by prescribed wall temperature [19-21] or for the boundary conditions of second kind, expressed by the prescribed wall heat flux $[22,23]$. A more realistic condition in many applications, however, will be temperature boundary conditions of third kind: the local wall heat flux is a linear function of the local temperature. Heat transfer in laminar region of a flat channel for the temperature boundary condition of third kind was explored by Javeri [24]. Javeri [25] investigated the influence of the temperature boundary condition of the third kind on the laminar heat transfer in the thermal entrance region of a rectangular channel. Later Zanchini
[26] analyzed the effect of viscous dissipation on mixed convection in a vertical channel with boundary conditions of third kind. Kumari and Nath [27] analyzed the effects of localized cooling/heating and injection/suction on mixed convection flow on a thin vertical cylinder.

The differential transformation method (DTM) was first applied in the engineering domain by Zhou [28]. The differential transform method is based on Taylor expansion. It constructs an analytical solution in the form of a polynomial. It is different from the traditional high order Taylor series method, which requires symbolic computation of the necessary derivatives of the data functions. The Taylor series method is computationally taken long time for large orders. The differential transform is an iterative procedure for obtaining analytic Taylor series solutions of differential equations. DTM has been successfully applied to solve many nonlinear problems arising in engineering, physics, mechanics, biology, etc. The differential transform method can overcome the restrictions and limitations of perturbation techniques so that it provides us with a possibility to analyze strongly nonlinear problems. Jang et al. [29] applied the two-dimensional differential transform method to the solution of partial differential equations. Kurnaz and Oturanç [30] applied DTM for solution of system of ordinary differential equations. Arikoglu and Ozkol [31] employed DTM on differential-difference equations. Ravi Kanth and Aruna [32] found the solution of singular two-point boundary value problems using differential transformation method. The method was successfully applied to various application problems [3335]. Very recently Rashidi et al. [36] applied the DTM to obtain approximate analytical solutions of combined free and forced (mixed) convection about inclined surfaces in a saturated porous medium.

Based on our review, a study on composite porous layer for mixed convection flow in a vertical channel with Robin boundary conditions is not found in the literature. Thus we concentrate on the case of mixed convection flow through a channel confined by parallel plate wall for composite porous matrix using Robin boundary conditions.

\section{Mathematical Formulation}

The geometry under consideration illustrated in figure. 1 consists of two infinite parallel plates maintained at equal or different constant temperatures extending in the $X$ and $Z$ directions. The region $-h_{1} / 2 \leq Y \leq 0$ is occupied by a fluid-saturated porous medium of permeability $\kappa_{1}$, density $\rho_{1}$, viscosity $\mu_{1}$, thermal conductivity $k_{1}$, and thermal expansion coefficient $\beta_{1}$, and the region $0 \leq Y \leq h_{2} / 2$ is occupied by another porous medium of permeability $\kappa_{2}$, density $\rho_{2}$, viscosity $\mu_{2}$, thermal conductivity $k_{2}$, and thermal expansion coefficient $\beta_{2}$. The fluids are assumed to have constant properties except the density in the buoyancy term in the momentum equation 
$\rho_{1}=\rho_{0}\left[1-\beta_{1}\left(T_{1}-T_{0}\right)\right]$ and $\rho_{2}=\rho_{0}\left[1-\beta_{2}\left(T_{2}-T_{0}\right)\right]$. A fluid rises in the channel driven by buoyancy forces. The transport properties of both fluids are assumed to be constant.

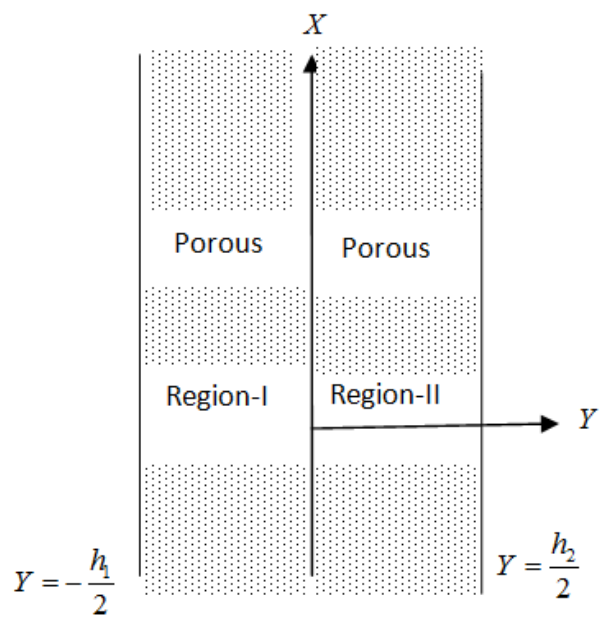

Fig 1: Physical configuration

We consider the fluids to be incompressible and the flow is steady, laminar, and fully developed. It is assumed that the only non-zero component of the velocity $\vec{q}$ are the $X$ component $U_{i}(i=1,2)$. Thus, as a consequence of the mass balance equation, one obtains

$$
\frac{\partial U_{i}}{\partial X}=0
$$

so that $U_{i}$ depends only on $Y$. The stream wise and the transverse momentum balance equations yields

$$
g \beta_{1}\left(T_{1}-T_{0}\right)-\frac{1}{\rho_{1}} \frac{\partial P}{\partial X}+v_{1} \frac{d^{2} U_{1}}{d Y^{2}}-\frac{v_{1}}{\kappa_{1}} U_{1}=0
$$

Region-II

$$
g \beta_{2}\left(T_{2}-T_{0}\right)-\frac{1}{\rho_{2}} \frac{\partial P}{\partial X}+v_{2} \frac{d^{2} U_{2}}{d Y^{2}}-\frac{\nu_{2}}{\kappa_{2}} U_{2}=0
$$

and $Y$-momentum balance equation in both the regions can be expressed as

$$
\frac{\partial P}{\partial Y}=0
$$

where $P=p+\rho_{0} g x$ (assuming $p_{1}=p_{2}=p \quad$ ) is the difference between the pressure and hydrostatic pressure. On account of (4) $P$ depends only on $X$ so that (2) and (3) can be rewritten as

Region-I

$$
T_{1}-T_{0}=\frac{1}{g \beta_{1} \rho_{1}} \frac{d P}{d X}-\frac{v_{1}}{g \beta_{1}} \frac{d^{2} U_{1}}{d Y^{2}}+\frac{v_{1}}{g \beta_{1} \kappa_{1}} U_{1}
$$

Region-II

$$
T_{2}-T_{0}=\frac{1}{g \beta_{2} \rho_{2}} \frac{d P}{d X}-\frac{v_{2}}{g \beta_{2}} \frac{d^{2} U_{2}}{d Y^{2}}+\frac{v_{2}}{g \beta_{2} \kappa_{2}} U_{2}
$$

From (5) and (6) one obtains

Region-I

$$
\begin{gathered}
\frac{\partial T_{1}}{\partial X}=\frac{1}{g \beta_{1} \rho_{1}} \frac{d^{2} P}{d X^{2}} \\
\frac{\partial T_{1}}{\partial Y}=-\frac{v_{1}}{g \beta_{1}} \frac{d^{3} U_{1}}{d Y^{3}}+\frac{v_{1}}{g \beta_{1} \kappa_{1}} \frac{d U_{1}}{d Y} \\
\frac{\partial^{2} T_{1}}{\partial Y^{2}}=-\frac{v_{1}}{g \beta_{1}} \frac{d^{4} U_{1}}{d Y^{4}}+\frac{v_{1}}{g \beta_{1} \kappa_{1}} \frac{d^{2} U_{1}}{d Y^{2}}
\end{gathered}
$$

Region-II

$$
\begin{gathered}
\frac{\partial T_{2}}{\partial X}=\frac{1}{g \beta_{2} \rho_{2}} \frac{d^{2} P}{d X^{2}} \\
\frac{\partial T_{2}}{\partial Y}=-\frac{v_{2}}{g \beta_{2}} \frac{d^{3} U_{2}}{d Y^{3}}+\frac{v_{2}}{g \beta_{2} \kappa_{2}} \frac{d U_{2}}{d Y} \\
\frac{\partial^{2} T_{2}}{\partial Y^{2}}=-\frac{v_{2}}{g \beta_{2}} \frac{d^{4} U_{2}}{d Y^{4}}+\frac{v_{2}}{g \beta_{2} \kappa_{2}} \frac{d^{2} U_{2}}{d Y^{2}}
\end{gathered}
$$

Both the walls of the channel will be assumed to have a negligible thickness and to exchange heat by convection with an external fluid. In particular, at $Y=-h_{1} / 2$ the external convection coefficient will be considered as uniform with the value $q_{1}$ and the fluid in the region $-h_{1} / 2 \leq Y \leq 0$ will be assumed to have a uniform reference temperature $T_{q_{1}}$. At $Y=h_{2} / 2$ the external convection coefficient will be considered as uniform with the value $q_{2}$ and the fluid in the region $0 \leq Y \leq h_{2} / 2$ will be supposed to have a uniform reference temperature $T_{q_{2}} \geq T_{q_{1}}$. Therefore, the boundary conditions on the temperature field can be expressed as

$$
\begin{gathered}
-\left.k_{1} \frac{\partial T_{1}}{\partial Y}\right|_{Y=-\frac{h_{1}}{2}}=q_{1}\left(T_{q_{1}}-T_{1}\left(X,-h_{1} / 2\right)\right) \\
-\left.k_{2} \frac{\partial T_{2}}{\partial Y}\right|_{Y=\frac{h_{2}}{2}}=q_{2}\left(T_{2}\left(X, h_{2} / 2\right)-T_{q_{2}}\right)
\end{gathered}
$$

On account of (8) and (11), (13) and (14) can be rewritten as

$$
\frac{d^{3} U_{1}}{d Y^{3}}-\frac{1}{\kappa_{1}} \frac{d U_{1}}{d Y}=\frac{g \beta_{1}}{k_{1} v_{1}} q_{1}\left(T_{q_{1}}-T_{1}\left(X,-h_{1} / 2\right)\right) \text { at } Y=-\frac{h_{1}}{2}
$$




$$
\frac{d^{3} U_{2}}{d Y^{3}}-\frac{1}{\kappa_{2}} \frac{d U_{2}}{d Y}=\frac{g \beta_{2}}{k_{2} V_{2}} q_{2}\left(T_{2}\left(X, h_{2} / 2\right)-T_{q_{2}}\right) \text { at } Y=\frac{h_{2}}{2}
$$

On account of (5) and (6), there exist a constant $A$ such that

$$
\frac{d P}{d X}=A
$$

For the problem under examination, the energy balance equation in the presence of viscous dissipation can be written as

Region-I

$$
\frac{d^{2} T_{1}}{\partial Y^{2}}=-\frac{v_{1}}{\alpha_{1} C_{p}}\left(\frac{d U_{1}}{d Y}\right)^{2}-\frac{v_{1}}{C_{p} \kappa_{1} \alpha_{1}} U_{1}^{2}
$$

Region-II

$$
\frac{d^{2} T_{2}}{\partial Y^{2}}=-\frac{v_{2}}{\alpha_{2} C_{p}}\left(\frac{d U_{2}}{d Y}\right)^{2}-\frac{v_{2}}{C_{p} \kappa_{2} \alpha_{2}} U_{2}^{2}
$$

From (9), (18), (12) and (19) allow one to obtain differential equations for $U_{i}$ namely

Region-I

$$
\frac{d^{4} U_{1}}{d Y^{4}}=g \beta_{1}\left(\frac{1}{g \beta_{1} \kappa_{1}} \frac{d^{2} U_{1}}{d Y^{2}}+\frac{1}{c_{p} \alpha_{1}}\left(\frac{d U_{1}}{d Y}\right)^{2}+\frac{U_{1}^{2}}{c_{p} \kappa_{1} \alpha_{1}}\right)
$$

\section{Region-II}

$$
\frac{d^{4} U_{2}}{d Y^{4}}=g \beta_{2}\left(\frac{1}{g \beta_{2} \kappa_{2}} \frac{d^{2} U_{2}}{d Y^{2}}+\frac{1}{c_{p} \alpha_{2}}\left(\frac{d U_{2}}{d Y}\right)^{2}+\frac{U_{2}^{2}}{c_{p} \kappa_{2} \alpha_{2}}\right)
$$

The boundary conditions on velocity are no-slip conditions, and those induced by boundary conditions on temperature. In addition, the continuity of velocity, shear stress, temperature and heat flux at the interface between the two porous layers are assumed as:

$$
U_{1}\left(-h_{1} / 2\right)=U_{2}\left(h_{2} / 2\right)=0
$$

together with (15) and (16) which on account of (5) and (6) can be rewritten as

$$
\begin{aligned}
\frac{d^{3} U_{1}}{d Y^{3}}-\frac{1}{\kappa_{1}} \frac{d U_{1}}{d Y}-\frac{q_{1}}{k_{1}} \frac{d^{2} U_{1}}{d Y^{2}} & =\frac{q_{1}}{k_{1}} \frac{g \beta_{1}}{V_{1}}\left(T_{q_{1}}-T_{0}\right)-\frac{q_{1}}{k_{1}} \frac{A}{\mu_{1}} \text { at } \\
Y & =-\frac{h_{1}}{2}
\end{aligned}
$$$$
\frac{d^{3} U_{2}}{d Y^{3}}-\frac{1}{\kappa_{2}} \frac{d U_{2}}{d Y}+\frac{q_{2}}{k_{2}} \frac{d^{2} U_{2}}{d Y^{2}}=\frac{q_{2}}{k_{2}} \frac{A}{\mu_{2}}-\frac{q_{2}}{k_{2}} \frac{g \beta_{2}}{v_{2}}\left(T_{q_{2}}-T_{0}\right) \text { at }
$$$$
Y=\frac{h_{2}}{2}
$$

$$
\begin{gathered}
U_{1}(0)=U_{2}(0), \mu_{1} \frac{d U_{1}(0)}{d Y}=\mu_{2} \frac{d U_{2}(0)}{d Y}, T_{1}(0)=T_{2}(0), \\
k_{1} \frac{d T_{1}(0)}{d Y}=k_{2} \frac{d T_{2}(0)}{d Y}
\end{gathered}
$$

Equations (20)-(24) determine the velocity distribution. They can be written in a dimensionless form by means of the following dimensionless parameters

$$
\begin{gathered}
u_{1}=\frac{U_{1}}{U_{0}^{(1)}}, u_{2}=\frac{U_{2}}{U_{0}^{(2)}}, y_{1}=\frac{Y_{1}}{D_{1}}, y_{2}=\frac{Y_{2}}{D_{2}}, G r=\frac{g \beta_{1} \Delta T D_{1}^{3}}{v_{1}^{2}}, \\
\operatorname{Re}=\frac{U_{0}^{(1)} D_{1}}{v_{1}}, B r=\frac{U_{0}^{(1)^{2}} \mu_{1}}{k_{1} \Delta T}, \Lambda=\frac{G r}{\operatorname{Re}}, \quad \theta_{1}=\frac{T_{1}-T_{0}}{\Delta T}, \\
\theta_{2}=\frac{T_{2}-T_{0}}{\Delta T}, R_{T}=\frac{T_{2}-T_{1}}{\Delta T}, D a_{1}=\frac{\kappa_{1}}{D_{1}^{2}}, D a_{2}=\frac{\kappa_{2}}{D_{2}^{2}}, \\
B i_{1}=\frac{h_{1} D_{1}}{k_{1}}, B i_{2}=\frac{h_{2} D_{2}}{k_{2}}, \quad s=\frac{B i_{1} B i_{2}}{B i_{1} B i_{2}+2 B i_{1}+2 B i_{2}}
\end{gathered}
$$

The reference velocity and the reference temperature are given by

$$
\begin{gathered}
U_{0}^{(1)}=-\frac{A D_{1}^{2}}{48 \mu_{1}}, \\
U_{0}^{(2)}=-\frac{A D_{2}^{2}}{48 \mu_{2}}, T_{0}=\frac{T_{q_{1}}+T_{q_{2}}}{2}+s\left(\frac{1}{B i_{1}}-\frac{1}{B i_{2}}\right)\left(T_{q_{2}}-T_{q_{1}}\right)
\end{gathered}
$$

Moreover, the temperature difference $\Delta T$ is given by $\Delta T=T_{q_{2}}-T_{q_{1}}$ if $T_{q_{1}}<T_{q_{2}}$. As a consequence, the dimensionless parameter $R_{T}$ can only take the values 0 or 1 . More precisely, the temperature difference ratio $R_{T}$ is equal to 1 for asymmetric heating i.e. $T_{q_{1}}<T_{q_{2}}$, while $R_{T}=0$ for symmetric heating i.e. $T_{q_{1}}=T_{q_{2}}$, respectively. Equation (17) implies that $A$ can be either positive or negative. If $A<0$, then $U_{0}^{i}, \operatorname{Re}$, and $\Lambda$ are negative, i.e. the flow is downward. On the other hand, if $A>0$, the flow is upward, so that $U_{0}^{i}, \operatorname{Re}$, and $\Lambda$ are positive. Using (25) and (26), (20)-(24) becomes

Region-I

$$
\frac{d^{4} u_{1}}{d y^{4}}-\frac{1}{D a_{1}} \frac{d^{2} u_{1}}{d y^{2}}=\Lambda B r\left(\left(\frac{d u_{1}}{d y}\right)^{2}+\frac{u_{1}^{2}}{D a_{1}}\right)
$$

Region-II

$$
\frac{d^{4} u_{2}}{d y^{4}}-\frac{1}{D a_{2}} \frac{d^{2} u_{2}}{d y^{2}}=\Lambda B r b h^{4} k n m\left(\left(\frac{d u_{2}}{d y}\right)^{2}+\frac{u_{2}^{2}}{D a_{2}}\right)
$$

The boundary and interface conditions becomes

$$
u_{1}(-1 / 4)=u_{2}(1 / 4)=0,
$$




$$
\begin{gathered}
\left(\frac{d^{2} u_{1}}{d y^{2}}+\frac{1}{D a_{1} B i_{1}} \frac{d u_{1}}{d y}-\frac{1}{B i_{1}} \frac{d^{3} u_{1}}{d y^{3}}\right)_{y=-1 / 4}=-48+\frac{R_{T} \Lambda s}{2}\left(1+\frac{4}{B i_{1}}\right) \\
\left(\frac{d^{2} u_{2}}{d y^{2}}+\frac{1}{B i_{2}} \frac{d^{3} u_{2}}{d y^{3}}-\frac{1}{B i_{2} D a_{2}} \frac{d u_{2}}{d y}\right)_{y=1 / 4}=-48-\frac{R_{T} \operatorname{sbn} \Lambda}{2}\left(1+\frac{4}{B i_{2}}\right) \\
u_{1}(0)=m h^{2} u_{2}(0), \frac{d u_{1}(0)}{d y}=h \frac{d u_{2}(0)}{d y}, \\
\left(\frac{d^{2} u_{1}}{d y^{2}}-\frac{u_{1}}{D a_{1}}\right)=\frac{1}{n b}\left(\frac{d^{2} u_{2}}{d y^{2}}-\frac{u_{2}}{D a_{2}}\right)+48\left(\frac{1}{n b}-1\right) \quad \text { at } Y=0 \\
\left(\frac{d^{3} u_{1}}{d y^{3}}-\frac{1}{D a_{1}} \frac{d u_{1}}{d y}\right)=\frac{1}{n b k h}\left(\frac{d^{3} u_{2}}{d y^{3}}-\frac{1}{D a_{2}} \frac{d u_{2}}{d y}\right) \text { at } Y=0
\end{gathered}
$$

\subsection{Basic Idea of Differential Transformation Method (DTM)}

If $u(y)$ is analytic in the time domain $\mathrm{T}$, then it will be differentiated continuously with respect to $y$ in the domain of interest. The differential transform of function $u(y)$ is defined as

$$
U(k)=\frac{1}{k !}\left[\frac{d^{k} u(y)}{d y^{k}}\right]_{y=0}
$$

where $u(y)$ is the original function and $U(k)$ is the transformed function which is called the T-function.

The differential inverse transform of $U(k)$ is defined as follows:

$$
u(y)=\sum_{k=0}^{\infty} U(k) y^{k}
$$

In real applications, the function $u(y)$ by a finite series of (31) can be written as

$$
u(y)=\sum_{k=0}^{n} U(k) y^{k}
$$

Table 1: The operations for the one-dimensional differential transform method.

\begin{tabular}{ll}
\hline Original function & Transformed function \\
\hline$y(x)=g(x) \pm h(x)$ & $Y(k)=G(k) \pm H(k)$ \\
$y(x)=\alpha g(x)$ & $Y(k)=\alpha G(k)$ \\
$y(x)=\frac{d g(x)}{d x}$ & $Y(k)=(k+1) G(k+1)$ \\
$y(x)=\frac{d^{2} g(x)}{d x^{2}}$ & $Y(k)=\sum_{l=0}^{k} G(l) H(k-l) G(k+2)$ \\
$y(x)=g(x) h(x)$ & $Y(k)=\delta(k-m)=\left\{\begin{array}{l}1, \text { if } k=m \\
0, \text { if } k \neq m\end{array}\right.$ \\
\hline$y(x)=x^{m}$
\end{tabular}

and (31) implies that $u(y)=\sum_{k=n+1}^{\infty} U(k) y^{k}$ and is neglected as it is small. Usually, the values of $n$ are decided by a convergence of the series coefficients. The fundamental mathematical operations performed by differential transform method are listed in Table 1.

\section{Solutions}

\subsection{Special Cases}

\subsubsection{Case of Negligible Viscous Dissipation $(\mathrm{Br}=0)$}

The solution of (27) and (28) using boundary and interface conditions in (29) in the absence of viscous dissipation term $(\mathrm{Br}=0)$ is given by

Region-I

$$
u_{1}=l_{1}+l_{2} y+l_{3} \cosh \left(M_{1} y\right)+l_{4} \sinh \left(M_{1} y\right)
$$

Region-II

$$
u_{2}=l_{5}+l_{6} y+l_{7} \cosh \left(M_{2} y\right)+l_{8} \sinh \left(M_{2} y\right)
$$

where $M_{1}=\left(1 / \sqrt{D a_{1}}\right), M_{2}=\left(1 / \sqrt{D a_{2}}\right)$

Using (29) in (5) and (6), the energy balance equations becomes

Region-I

$$
\theta_{1}=\frac{1}{\Lambda}\left(-48-\frac{d^{2} u_{1}}{d y^{2}}+\frac{u_{1}}{D a_{1}}\right)
$$

Region-II

$$
\theta_{2}=\frac{1}{\Lambda b n}\left(-48-\frac{d^{2} u_{2}}{d y^{2}}+\frac{u_{2}}{D a_{2}}\right)
$$

Using the expressions obtained in (33) and (34) the energy balance (35) and (36) becomes

Region-I

$$
\theta_{1}=\frac{1}{\Lambda}\left(-48+\frac{\left(l_{1}+l_{2} y\right)}{D a_{1}}+r_{1} \cosh \left(M_{1} y\right)+r_{2} \sinh \left(M_{1} y\right)\right)
$$

Region-II

$$
\theta_{2}=\frac{1}{\Lambda b n}\left(-48+\frac{\left(l_{5}+l_{6} y\right)}{D a_{2}}+r_{3} \cosh \left(M_{2} y\right)+r_{4} \sinh \left(M_{2} y\right)\right)
$$

\subsubsection{Case of Negligible Buoyancy Force $(\Lambda=0)$}

The solution of (27) and (28) can be obtained when buoyancy forces are negligible $(\Lambda=0)$ and viscous dissipation is dominating ( $B r \neq 0)$, so that purely forced convection occurs. For this case, solutions of (27) and (28), using the boundary and interface conditions given by (29), the velocities are given by

Region-I 


$$
u_{1}=f_{1}+f_{2} y+f_{3} \cosh \left(M_{1} y\right)+f_{4} \sinh \left(M_{1} y\right)
$$

Region-II

$$
u_{2}=f_{5}+f_{6} y+f_{7} \cosh \left(M_{2} y\right)+f_{8} \sinh \left(M_{2} y\right)
$$

The energy balance (18) and (19) in non-dimensional form can also be written as

Region-I

$$
\frac{d^{2} \theta_{1}}{d y^{2}}=-B r\left(\left(\frac{d u_{1}}{d y}\right)^{2}+\frac{u_{1}^{2}}{D a_{1}}\right)
$$

Region-II

$$
\begin{gathered}
\frac{d^{2} \theta_{2}}{d y^{2}}=-B r k m h^{4}\left(\left(\frac{d u_{2}}{d y}\right)^{2}+\frac{u_{2}^{2}}{D a_{2}}\right) \\
\theta_{1}=-B r\left(p_{10} \cosh \left(2 M_{1} y\right)+p_{11} \sinh \left(2 M_{1} y\right)+p_{12} \cosh \left(M_{1} y\right)+p_{13} \sinh \left(M_{1} y\right)+\right. \\
\left.p_{14} y \cosh \left(M_{1} y\right)+p_{15} y \sinh \left(M_{1} y\right)+p_{16} y^{4}+p_{17} y^{3}+p_{18} y^{2}\right)+c_{1} y+c_{2}
\end{gathered}
$$

Using (39) and (40), solving (41) and (42) we obtain Region-I

Region-II

$$
\begin{aligned}
\theta_{2}= & -\operatorname{Brmkh}^{4}\left(p_{28} \cosh \left(2 M_{2} y\right)+p_{29} \sinh \left(2 M_{2} y\right)+p_{30} \cosh \left(M_{2} y\right)+p_{31} \sinh \left(M_{2} y\right)\right. \\
& \left.+p_{32} y \cosh \left(M_{2} y\right)+p_{33} y \sinh \left(M_{2} y\right)+p_{34} y^{4}+p_{35} y^{3}+p_{36} y^{2}\right)+c_{3} y+c_{4}
\end{aligned}
$$

\subsection{Perturbation Method (PM)}

\subsubsection{Combined Effects of Buoyancy Forces and Viscous Dissipation}

We solve (27) and (28) using the perturbation method with a dimensionless parameter $|\mathcal{E}|(<<1)$ defined as

$$
\varepsilon=\Lambda B r
$$

which does not depend on the reference temperature difference $\Delta T$. To this end the solutions are assumed in the form

$$
u(y)=u_{0}(y)+\varepsilon u_{1}(y)+\varepsilon^{2} u_{2}(y)+\ldots=\sum_{n=0}^{\infty} \varepsilon^{n} u_{n}(y)
$$

Substituting (47) in (27) and (28) and equating the coefficients of like powers of $\varepsilon$ to zero, we obtain the zero and first order equations as follows:

Region-I

Zero-order equations

$$
\frac{d^{4} u_{10}}{d y^{4}}-\frac{1}{D a_{1}} \frac{d^{2} u_{10}}{d y^{2}}=0
$$

First-order equations

$$
\frac{d^{4} u_{11}}{d y^{4}}-\frac{1}{D a_{1}} \frac{d^{2} u_{11}}{d y^{2}}=\left(\frac{d u_{10}}{d y}\right)^{2}+\frac{u_{10}^{2}}{D a_{1}}
$$

Region-II

Zero-order equations

$$
\frac{d^{4} u_{20}}{d y^{4}}-\frac{1}{D a_{2}} \frac{d^{2} u_{20}}{d y^{2}}=0
$$

First-order equations

$$
\frac{d^{4} u_{21}}{d y^{4}}-\frac{1}{D a_{2}} \frac{d^{2} u_{21}}{d y^{2}}=z_{1}\left(\left(\frac{d u_{20}}{d y}\right)^{2}+\frac{u_{20}^{2}}{D a_{2}}\right)
$$

The corresponding boundary and interface conditions given by (29) for the zeroth and first order reduces to

Zero-order

$$
\begin{gathered}
u_{10}(-1 / 4)=u_{20}(1 / 4)=0, u_{10}(0)=m h^{2} u_{20}(0), \\
\frac{d u_{10}(0)}{d y}=h \frac{d u_{20}(0)}{d y},
\end{gathered}
$$

$$
\begin{aligned}
& \left(\frac{d^{2} u_{10}}{d y^{2}}-\frac{u_{10}}{D a_{1}}\right)=\frac{1}{n b}\left(\frac{d^{2} u_{20}}{d y^{2}}-\frac{u_{20}}{D a_{2}}\right)+48\left(\frac{1}{n b}-1\right) \text { at } y=0, \\
& \left(\frac{d^{3} u_{10}}{d y^{3}}-\frac{1}{D a_{1}} \frac{d u_{10}}{d y}\right)=\frac{1}{z_{2}}\left(\frac{d^{3} u_{20}}{d y^{3}}-\frac{1}{D a_{2}} \frac{d u_{20}}{d y}\right) \quad \text { at } y=0, \\
& \left(\frac{d^{2} u_{10}}{d y^{2}}+\frac{1}{D a_{1} B i_{1}} \frac{d u_{10}}{d y}-\frac{1}{B i_{1}} \frac{d^{3} u_{10}}{d y^{3}}\right)_{y=-1 / 4}=-48+\frac{R_{T} \Lambda s}{2}\left(1+\frac{4}{B i_{1}}\right),
\end{aligned}
$$


$\left(\frac{d^{2} u_{20}}{d y^{2}}+\frac{1}{B i_{2}} \frac{d^{3} u_{20}}{d y^{3}}-\frac{1}{B i_{2} D a_{2}} \frac{d u_{20}}{d y}\right)_{y=1 / 4}=-48-\frac{R_{T} \Lambda s b n}{2}\left(1+\frac{4}{B i_{2}}\right)$

First-order

$$
\begin{aligned}
& u_{11}(-1 / 4)=u_{21}(1 / 4)=0, \quad u_{11}(0)=m h^{2} u_{21}(0), \\
& \frac{d u_{11}(0)}{d y}=h \frac{d u_{21}(0)}{d y} \\
& \left(\frac{d^{2} u_{11}}{d y^{2}}-\frac{u_{11}}{D a_{1}}\right)=\frac{1}{n b}\left(\frac{d^{2} u_{21}}{d y^{2}}-\frac{u_{21}}{D a_{2}}\right) \text { at } y=0, \\
& \left(\frac{d^{3} u_{11}}{d y^{3}}-\frac{1}{D a_{1}} \frac{d u_{11}}{d y}\right)=\frac{1}{z_{2}}\left(\frac{d^{3} u_{21}}{d y^{3}}-\frac{1}{D a_{2}} \frac{d u_{21}}{d y}\right) \text { at } y=0 \\
& \begin{aligned}
u_{11}= & H_{9}+H_{10} y+H_{11} \cosh \left(M_{1} y\right)+H_{12} \sinh \left(M_{1} y\right)+k_{10} \cosh \left(2 M_{1} y\right)+ \\
& k_{11} \sinh \left(2 M_{1} y\right)+k_{12} y \sinh \left(M_{1} y\right)+k_{13} y \cosh \left(M_{1} y\right)+k_{14} y^{2} \cosh \left(M_{1} y\right)+ \\
& k_{15} y^{2} \sinh \left(M_{1} y\right)+k_{16} y^{4}+k_{17} y^{3}+k_{18} y^{2} \\
u_{21}= & H_{13}+H_{14} y+H_{15} \cosh \left(M_{2} y\right)+H_{16} \sinh \left(M_{2} y\right)+t_{1}\left(k_{28} \cosh \left(2 M_{2} y\right)+\right. \\
& k_{29} \sinh \left(2 M_{2} y\right)+k_{30} y \sinh \left(M_{2} y\right)+k_{31} y \cosh \left(M_{2} y\right)+k_{32} y^{2} \cosh \left(M_{2} y\right)+ \\
& \left.k_{33} y^{2} \sinh \left(M_{2} y\right)+k_{34} y^{4}+k_{35} y^{3}+k_{36} y^{2}\right)
\end{aligned}
\end{aligned}
$$

Using velocities given by (54)-(57), the expressions for energy balance (35) and (36) becomes

Region-I

$$
\begin{aligned}
\theta_{1}= & \frac{1}{\Lambda}\left(-48-\varepsilon\left(3 M_{1}^{2} k_{10} \cosh \left(2 M_{1} y\right)+3 M_{1}^{2} k_{11} \sinh \left(2 M_{1} y\right)+2 M_{1} k_{12} \cosh \left(M_{1} y\right)\right.\right. \\
& +2 M_{1} k_{13} \sinh \left(M_{1} y\right)+k_{14}\left(4 M_{1} y \sinh \left(M_{1} y\right)+2 \cosh \left(M_{1} y\right)\right) \\
& +k_{15}\left(4 M_{1} y \cosh \left(M_{1} y\right)+2 \sinh \left(M_{1} y\right)\right)+M_{1}^{2} k_{16} y^{4}+M_{1}^{2} k_{17} y^{3}+\left(12 k_{16}+M_{1}^{2} k_{18}\right) y^{2} \\
& \left.\left.+\left(6 k_{17}+M_{1}^{2} H_{10}\right) y+\left(2 k_{18}+M_{1}^{2} H_{9}\right)\right)+M_{1}^{2}\left(H_{1}+H_{2} y\right)\right)
\end{aligned}
$$

Region-II

$$
\begin{aligned}
\theta_{2}= & \frac{1}{\Lambda b n}\left(-48-\varepsilon\left(z _ { 1 } \left(3 M_{2}^{2} k_{28} \cosh \left(2 M_{2} y\right)+3 M_{2}{ }^{2} k_{29} \sinh \left(2 M_{2} y\right)+2 M_{2} k_{30} \cosh \left(M_{2} y\right)\right.\right.\right. \\
& +2 M_{2} k_{31} \sinh \left(M_{2} y\right)+k_{32}\left(4 M_{2} y \sinh \left(M_{2} y\right)+2 \cosh \left(M_{2} y\right)\right)+k_{33}\left(4 M_{2} y \cosh \left(M_{2} y\right)\right. \\
& \left.\left.+2 \sinh \left(M_{2} y\right)\right)+M_{2}{ }^{2}\left(k_{34} y^{4}+k_{35} y^{3}\right)\right)+z_{1}\left(12 k_{34}+M_{2}{ }^{2} k_{36}\right) y^{2}+6 z_{1} k_{35} y+2 z_{1} k_{36} \\
& \left.\left.+M_{2}^{2}\left(H_{13}+H_{14} y\right)\right)+M_{2}{ }^{2}\left(H_{5}+H_{6} y\right)\right)
\end{aligned}
$$

\subsection{Solution with Differential Transform Method}

Now Differential Transformation method has been applied for solving (27) and (28).Taking the differential transformation of (27) and (28) with respect to $k$, and following the process as given in Table 1 yields: 


$$
\begin{gathered}
U(r+4)=\frac{1}{(r+1)(r+2)(r+3)(r+4)}\left(\frac{1}{D a_{1}}(r+1)(r+2) U(r+2)\right. \\
\left.+\Lambda B r\left(\sum_{s=0}^{r}(r-s+1)(s+1) U(r-s+1) U(s+1)+\frac{1}{D a_{1}} \sum_{s=0}^{r} U(s) U(r-s)\right)\right) \\
V(r+4)=\frac{1}{(r+1)(r+2)(r+3)(r+4)}\left(\frac{(r+1)(r+2)}{D a_{2}} V(r+2)\right. \\
\left.+\Lambda B r b n k m h^{4}\left(\sum_{s=0}^{r}(r-s+1)(s+1) V(r-s+1) V(s+1)+\frac{1}{D a_{2}} \sum_{s=0}^{r} V(r-s) V(s)\right)\right)
\end{gathered}
$$

The differential transform of the initial conditions are as follows

$$
\begin{gathered}
U(0)=c_{1}, U(1)=c_{2}, U(2)=\frac{c_{3}}{2}, U(3)=\frac{c_{4}}{6}, V(0)=\frac{c_{1}}{m h^{2}}, V(1)=\frac{c_{2}}{h}, \\
V(2)=n b\left(c_{3}-48\left(\frac{1}{n b}-1\right)-\frac{c_{1}}{D a_{1}}+\frac{c_{1}}{D a_{2} n b m h^{2}}\right), V(3)=\left(c_{4}-c_{2} / D a_{2}\right) n b k h+c_{2} / h D a_{2}
\end{gathered}
$$

where $U(r)$ and $V(r)$ are the transformed versions of $u_{1}(y)$ and $u_{2}(y)$ respectively.

Using the conditions as given in (62), one can evaluate the unknowns $c_{1}, c_{2}, c_{3}$, and $c_{4}$. By using the DTM and the transformed boundary conditions, above equations that finally leads to the solution of a system of algebraic equations.

A Nusselt number can be defined at each boundary, as follows:

$$
\begin{gathered}
N u_{1}=\left.\frac{2\left(h_{1}+h_{2}\right)}{R_{T}\left[T_{2}\left(h_{2} / 2\right)-T_{1}\left(-h_{1} / 2\right)\right]+\left(1-R_{T}\right) \Delta T} \frac{d T_{1}}{d Y}\right|_{Y=-h_{1} / 2} \\
N u_{2}=\left.\frac{2\left(h_{1}+h_{2}\right)}{R_{T}\left[T_{2}\left(h_{2} / 2\right)-T_{1}\left(-h_{1} / 2\right)\right]+\left(1-R_{T}\right) \Delta T} \frac{d T_{2}}{d Y}\right|_{Y=h_{2} / 2}
\end{gathered}
$$

By employing (26), in (64) can be written as

$$
\begin{gathered}
N u_{1}=\left.\frac{(1+h)}{R_{T}\left[\theta_{2}(1 / 4)-\theta_{1}(-1 / 4)\right]+\left(1-R_{T}\right)} \frac{d \theta_{1}}{d y}\right|_{y=-1 / 4} \\
N u_{2}=\left.\frac{(1+1 / h)}{R_{T}\left[\theta_{2}(1 / 4)-\theta_{1}(-1 / 4)\right]+\left(1-R_{T}\right)} \frac{d \theta_{2}}{d y}\right|_{y=1 / 4} .
\end{gathered}
$$

All the constants appeared in the above equations are not presented due to want of space.

\section{Results and Discussion}

The governing (28) and (29) subject to the boundary conditions (30) have been solved analytically by regular perturbation method valid for small values of perturbation parameter $\varepsilon$. However this condition is relaxed by finding the solutions valid for all values of $\varepsilon$ by Differential Transform method which is a semi-analytical method. Both the cases of asymmetric $\left(R_{T}=1\right)$ and symmetric $\left(R_{T}=0\right)$ wall heating conditions are considered and the results are shown graphically in Figs. 2-13 for equal and unequal Biot numbers. When $\mathrm{Br}=0$ for equal Biot numbers, there is a flow reversal for $\Lambda=400$ at the left wall and for $\Lambda=-400$ at the right wall as seen in Fig. 2. Further Fig. 2 also display the result that as $\sigma$ increases velocity decreases in both the regions for all values of $\Lambda$. The nondimensional temperature field $\theta$ is evaluated for different values of Brinkman number in the case of negligible buoyancy force which corresponds to $\Lambda=0$ for equal and unequal Biot numbers in Figs. $3 a$ and $3 b$ respectively. The temperature field is linear in the absence of mixed convection parameter $\Lambda$ and viscous dissipation $(B r=0)$ indicating that the heat transfer is purely by conduction for both equal and unequal Biot numbers. The temperature field increases with increase in Brinkman number for both equal and unequal Biot numbers. However the nature of profiles for equal and unequal Biot numbers is different at the cold plate. One should realize that this nature is due to the fact that when $B i_{1}=0.1, B i_{2}=10$, one obtains $T(-L / 2)>T(L / 2)$ (following Zanchini, [26]). The effect of $\Lambda$ and $B r$ for two fluid model is the similar result observed by Zanchini [26] and Barletta [37] using asymmetric wall heating conditions for one fluid model. To compare the present results for one fluid model the values of ratios of viscosity, width and conductivity are fixed as 1 . 


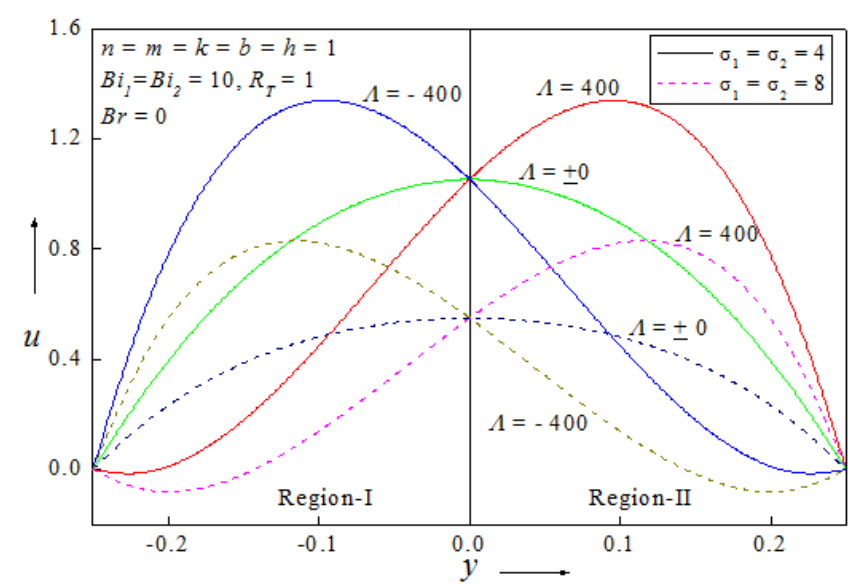

Fig.2: Plots of u versus y for different values of $\Lambda$.

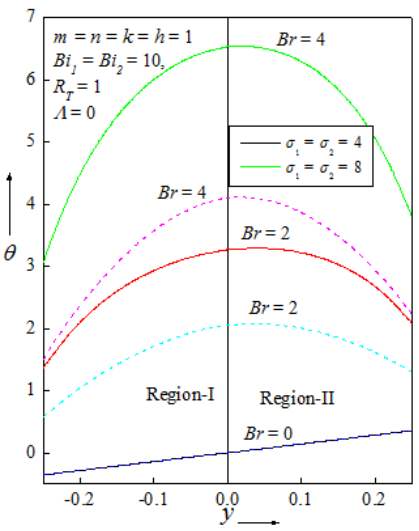

Fig.3a: Plots of $\theta$ versus y for different values of $B$ r.

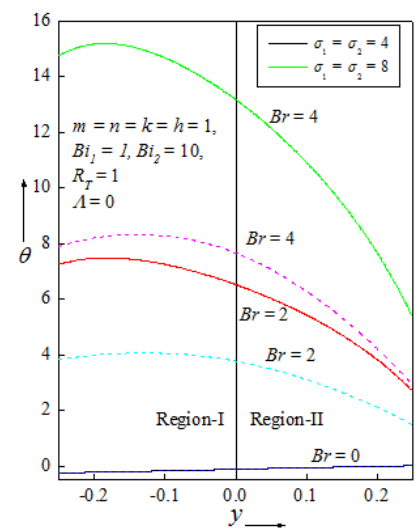

Fig.3b: Plots of $\theta$ versus $y$ for different values of $B$.

The combined effects of $\Lambda$ and $B r$ on the velocity and temperature fields $u$ and $\theta$ for different values of perturbation parameter $\varepsilon$ is shown in Figs. $4 \mathrm{a}$ and $4 \mathrm{~b}$ respectively for equal Biot numbers. For upward flow $(\Lambda>0, \varepsilon>0)$ velocity and temperature are increasing functions of $\varepsilon$. For downward flow $(\Lambda<0, \varepsilon<0)$ velocity is a decreasing function of $\varepsilon$ whereas temperature is an increasing function of $\varepsilon$. It is also observed from Fig. 4a that flow reversal occurs at the left wall for buoyancy assisting flow and at the right wall for buoyancy opposing flow. This nature is due the reason that increase in perturbation parameter $\varepsilon(\varepsilon>0)$ implies the enhancement of viscous dissipation which results in higher values of temperature and hence enhances the buoyancy forces also. Therefore velocity increases for $\Lambda>0$ and decreases for $\Lambda<0$. Figures $4 \mathrm{a}$ and $4 \mathrm{~b}$ also reveals that the solutions obtained by PM and DTM agree very well for small values of $\varepsilon$ and the difference is large as $\varepsilon$ increases. The effect of $\Lambda$ and $\varepsilon$ on the flow is the similar result obtained by Prathap Kumar et al. [17] for isothermal boundary conditions for composite porous medium and by Umavathi and Santhosh [38] for one fluid model.

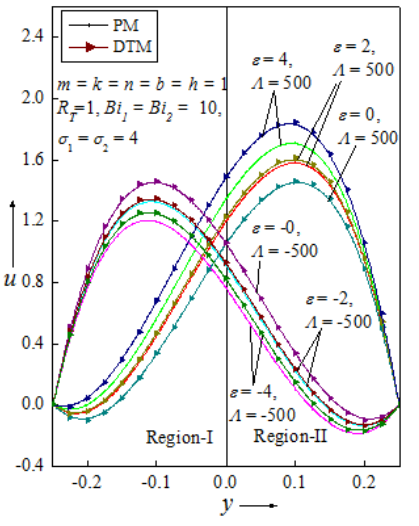

Fig.4a: Plots of $u$ versus y for different values of $\varepsilon$

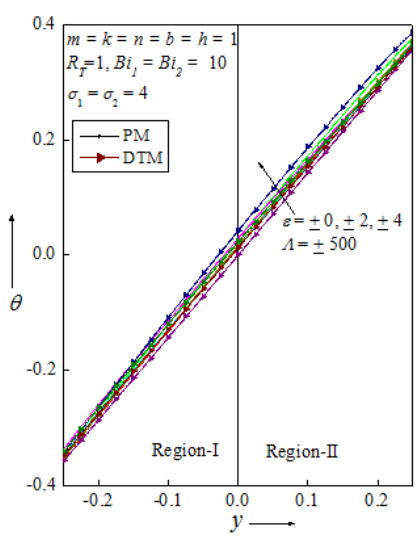

Fig.4b: Plots of $\theta$ versus y for different values of $\varepsilon$.
Figures $5 \mathrm{a}$ and $5 \mathrm{~b}$ are the plots of velocity and temperature for different values of porous parameter $\sigma\left(=\sigma_{1}=\sigma_{2}\right)$ for equal Biot numbers. As the porous parameter $\sigma$ increases both the velocity and temperature decreases in both the regions which is a classical result. One can infer this result as, the frictional drag resistance against the flow becomes pronounced for large values of $\sigma$ and hence the flow is reduced (for simplicity the porous parameter $\sigma$ is considered to be equal in both the regions). The effect of $\sigma$ on the flow was the similar result obtained by Prathap Kumar et al. [17] for isothermal boundary conditions and by Umavathi and Santhosh [38] for one fluid model using boundary conditions of third kind.

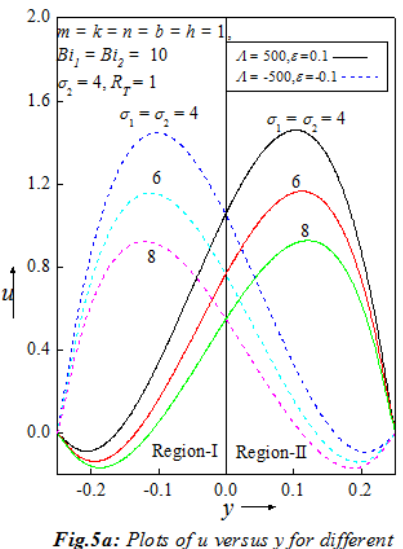

values of $\sigma_{1}=\sigma_{2}$

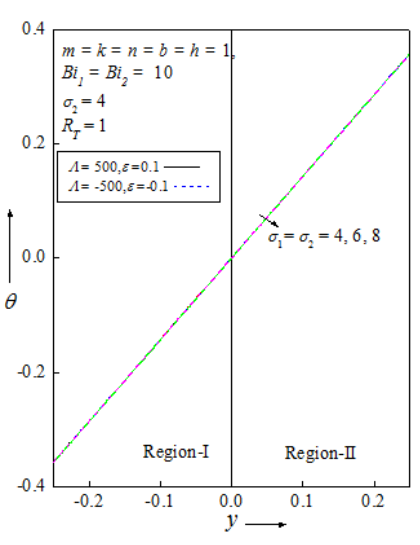

Fig.5b: Plots of $\theta$ versus $y$ for different values of $\sigma_{1}=\sigma_{2}$.
The effect of viscosity ratio $m\left(=\mu_{1} / \mu_{2}\right)$ on the velocity and temperature fields is shown in Figs. 6a and $6 \mathrm{~b}$ respectively for equal Biot numbers. As the viscosity ratio $m$ increases, the velocity increases in region-I and decreases in region-II. The viscosity ratio $m$ is taken as the ratio of viscosity of the fluid in region-I to the viscosity of the fluid in region-II. Physically, the values of $m>1$ implies the viscosity of the fluid in region-II is larger than the viscosity of the fluid in region-I. It is seen from Fig. $6 \mathrm{a}$ that for values of $m>1$ the magnitude of the velocity is less in region-II when compared to region-I. For values of $m=1$ (both the fluids in region-I and region-II have the same viscosity) show almost symmetric profiles. However the profile in region-I and region-II look different because 
of the value of $\Lambda$ chosen to be 500. Further there is a continuity of velocity at the interface for $m=1$ and sudden jump at the interface for values of $m>1$. The fall at the interface for values of $m>1$ is due to the nature of the condition imposed at the interface. Figure $6 \mathrm{~b}$ shows almost no variation for the effects of $m$ on the temperature. As the porous parameter $\sigma\left(=\sigma_{1}=\sigma_{2}\right)$ increases velocity decreases in both regions. However the effect of $\sigma$ is not effective on the temperature field as seen in Fig. 6 b.

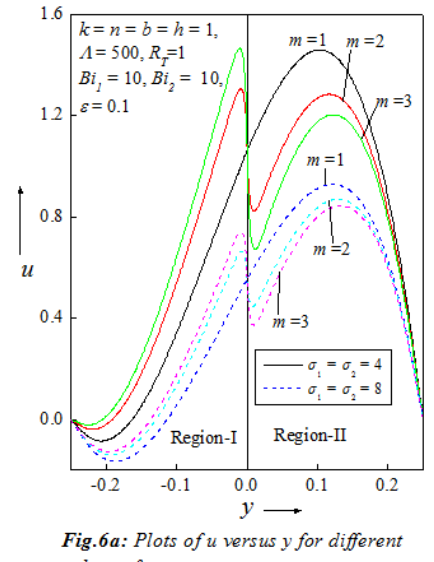

values of $m$.

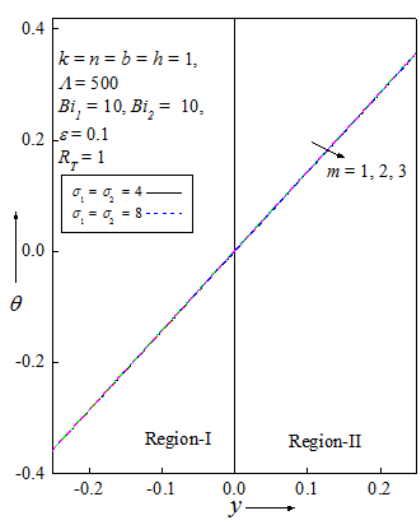

Fig.6b: Plots of $\theta$ versus $y$ for different values of $m$.
The effect of width ratio $h\left(h_{2} / h_{1}\right)$ on the flow field is shown in Figs. $7 \mathrm{a}$ and $7 \mathrm{~b}$ respectively. The velocity and temperature decreases in both regions as the width ratio $h$ increases. Physically, $h$ increases imply width of the fluid in region-I is wider than that of the fluid in region-II. The magnitude of suppression is significant in region-I when compared to region-II. Further from Fig. 7a one can also explore that as $\sigma\left(=\sigma_{1}=\sigma_{2}\right)$ increases velocity decreases in both regions. Figure $7 \mathrm{~b}$ also tells that the effect of $\sigma$ is not effective on the temperature field.

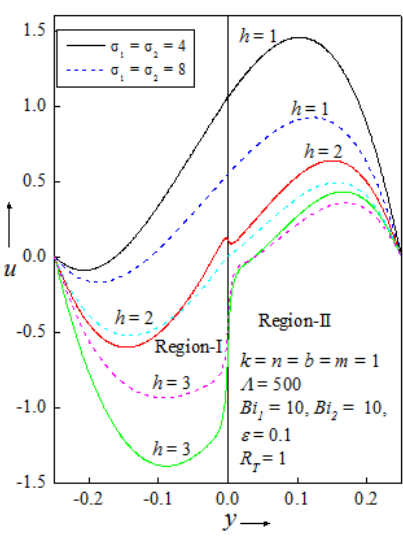

Fig.7a: Plots of $u$ versus $y$ for different values of $h$.

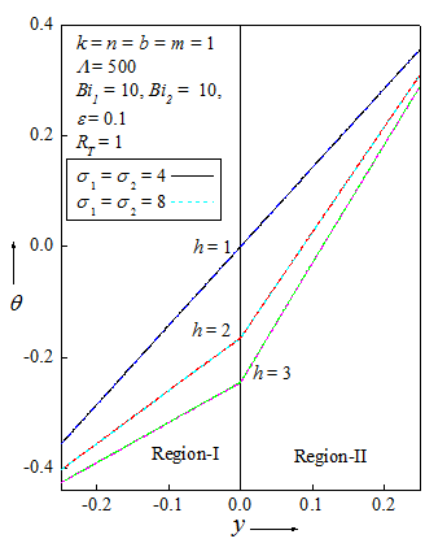

Fig. 7b: Plots of $\theta$ versus $y$ for different values of $h$.
The velocity and temperature fields for variations of conductivity ratio $k\left(k_{1} / k_{2}\right)$ are seen in Figs. $8 \mathrm{a}$ and $8 \mathrm{~b}$ respectively for equal Biot numbers. As $k$ increases both the velocity and temperature decreases in both regions. Values of $k>1$ implies the thermal conductivity in regionII is greater than the thermal conductivity in region-I.
Further as $\sigma$ increases velocity decreases in both the regions and is not effective on the temperature field.

The effect of $\Lambda$ and $\varepsilon$ for unequal Biot numbers on the velocity and temperature field is displayed in Figs. 9a and $9 \mathrm{~b}$ respectively. It is observed that for unequal Biot numbers there is no flow reversal at both the cold and hot walls for upward and downward flows when compared with equal Biot numbers. Further the nature of temperature profiles for unequal Biot numbers is more distinguishable at the cold wall when compared with equal Biot numbers. However the effect of $\Lambda$ and $\varepsilon$ for unequal Biot numbers is similar to the equal Biot numbers. Comparing Figs. $4 \mathrm{a}, \mathrm{b}$ (equal Biot numbers) and Figs. 9a,b (unequal Biot numbers) one can explore the result that the effect of $\Lambda$ on $u$ and $\theta$ becomes stronger if either $B i_{1}$ or $B i_{2}$ becomes stronger.

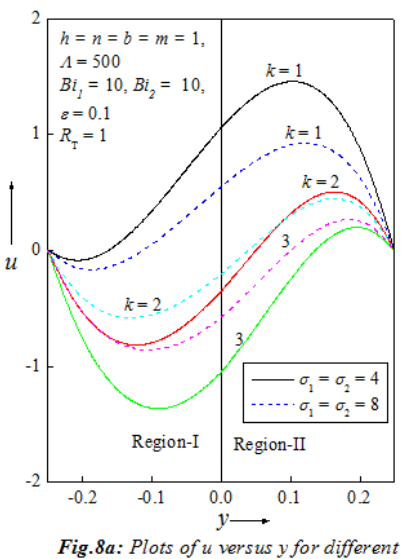
values of $k$

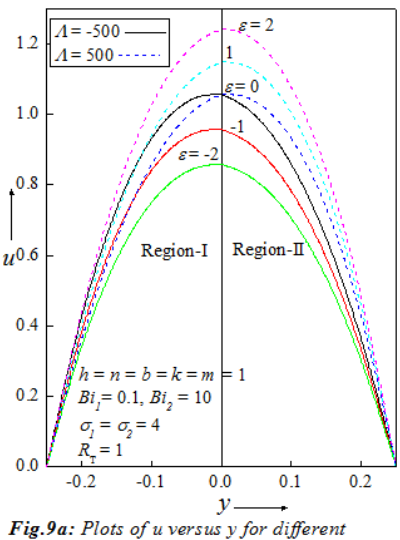

Fig.9a: Plots
values of 8

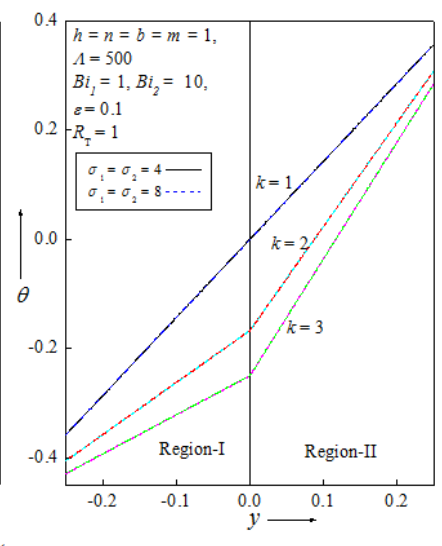

Fig.8b: Plots of $\theta$ versus $y$ for different values of $k$.

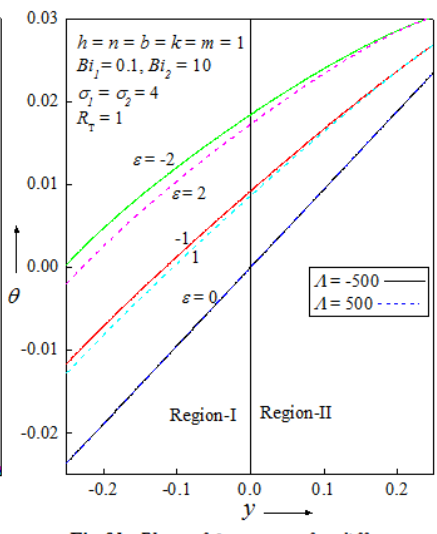

Fig.9b: Plots of $\theta$ versus $y$ for different values of $\varepsilon$.
The effect of porous parameter $\sigma\left(=\sigma_{1}=\sigma_{2}\right)$ for unequal Biot numbers on the flow field is depicted in Figs. 10a,b. The effect of $\sigma$ on the flow for unequal Biot numbers shows the similar nature as that on equal Biot numbers (Fig. 5a, b). That is as $\sigma$ increases velocity decreases in both the regions for both buoyancy assisting and buoyancy opposing flows. The effect of $\sigma$ is not changing the temperature field as seen Fig. 10b. The effect of $\sigma$ on the flow was the similar result obtained by Umavathi and Santosh [38] for unequal Biot numbers for one fluid model.

Figures $11 \mathrm{a}, \mathrm{b}$ and $12 \mathrm{a}, \mathrm{b}$ are the plots of $u$ and $\Lambda \theta$ for 
symmetric wall heating conditions considering equal and unequal Biot numbers. These figures show that $u$ and $\Lambda \theta$ are increasing functions of $\varepsilon$ for both equal and unequal Biot numbers. Further the velocity profiles are symmetric in nature for both equal and unequal Biot numbers (Figs. 11a and 12a). The temperature profiles at the cold wall shows different nature for equal and unequal Biot numbers (Figs. 11b and 12b). The similar nature was also observed for asymmetric wall heating conditions. The reason for this nature is explained above for equal Biot numbers. One can infer that symmetric wall heating conditions also the effect of $u$ and $\Lambda \theta$ is stronger if either $B i_{1}$ or $B i_{2}$ becomes smaller.

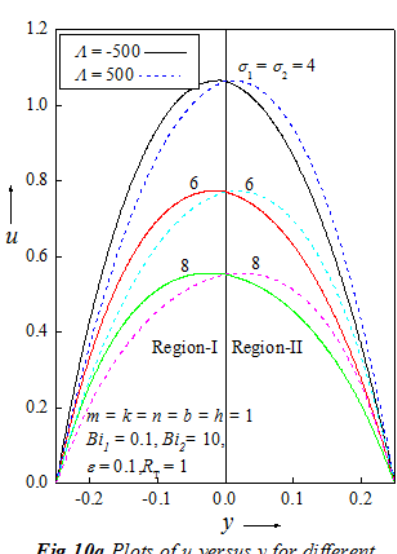

Fig.10a Plots of $u$ versus y for different values of $\sigma_{1}=\sigma_{2}$.

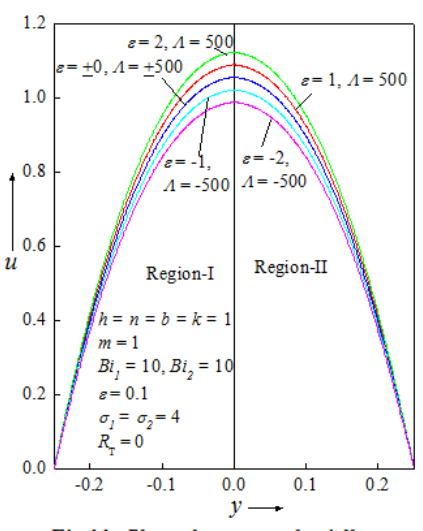

Fig.11a Plots of u versus y for different values of $\varepsilon$.

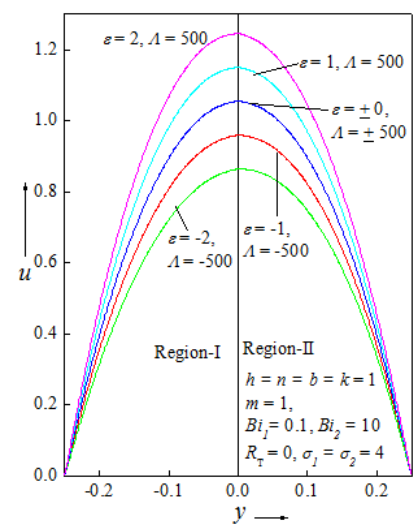

Fig.12a Plots of $u$ versus $y$ for different values of $\varepsilon$.

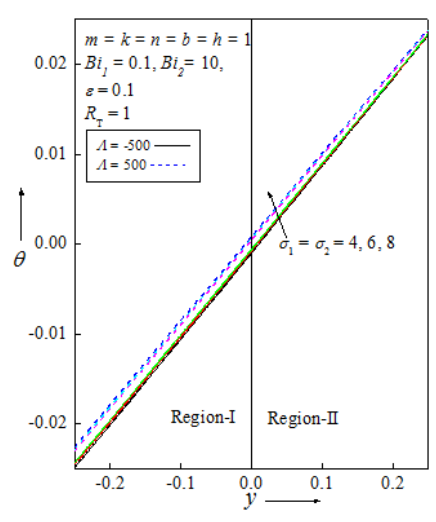

Fig.10b Plots of $\theta$ versus y for different values of $\sigma_{1}=\sigma_{2}$

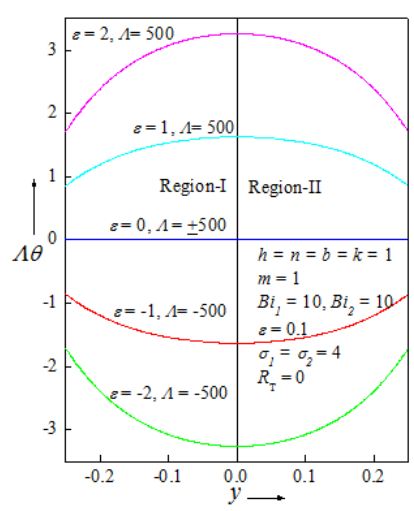

Fig.11b Plots of $\theta$ versus $y$ for different values of $\varepsilon$.

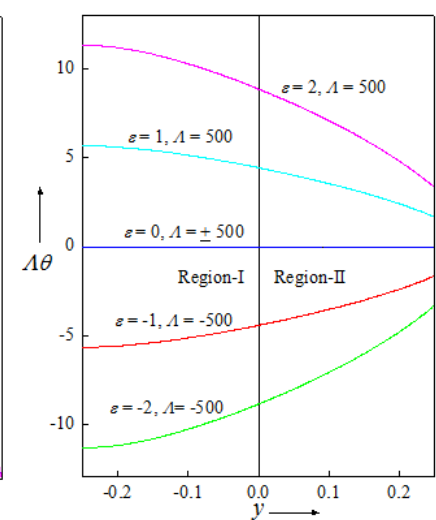

Fig.12b: Plots of $\theta$ versus $y$ for different values of $\varepsilon$.
The rate of heat transfer at both the walls for variation of $|\varepsilon|$ is shown in Fig. 13. The Nusselt number at the cold wall is a decreasing function of mixed convection parameter $\Lambda$ for upward flow and increasing function of $\Lambda$ for downward flow. The rate of heat transfer is more for smaller values of $\Lambda$ at the left wall. The Nusselt number is an increasing function of mixed convection parameter $\Lambda$ for downward flow at the hot wall and decreasing function of $\Lambda$ for upward flow. The flow nature of Nusselt number on $|\varepsilon|$ is the similar result obtained by Umavathi and Santhosh [38] for one fluid model.

It is observed from Fig. 14 that the Nusselt number at the cold wall is a decreasing function of porous parameter $\sigma$ for both upward and downward flows. Reversal effect is observed at the hot wall. That is to say that Nusselt number increases as $\sigma$ increases at the hot wall for both upward and downward flows.
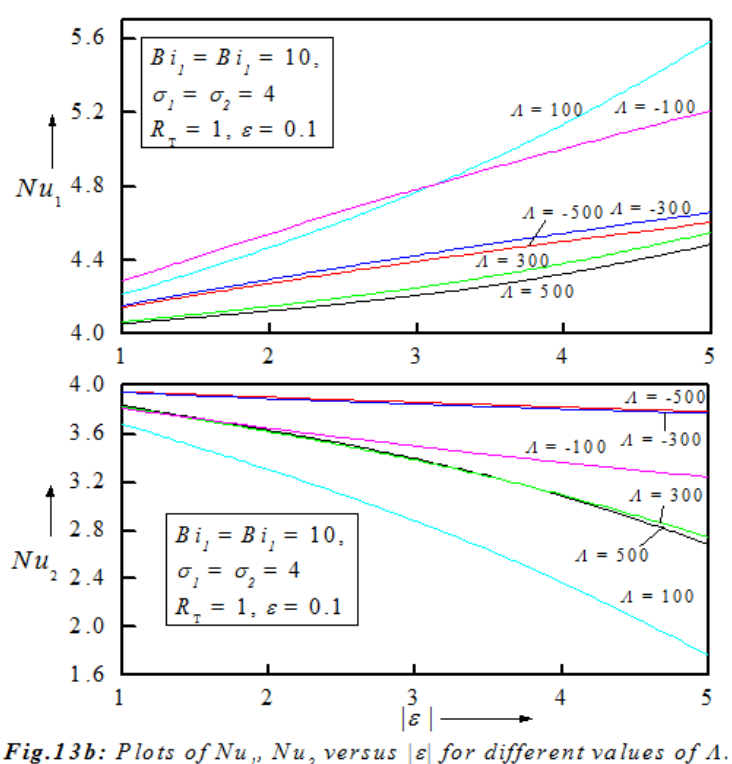

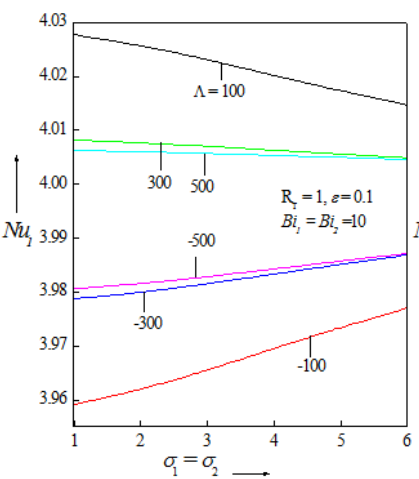

Fig.14a: Plots of $\mathrm{Nu}_{1}$ versus $\sigma_{1}=\sigma_{2}$ for different values of $\Lambda$

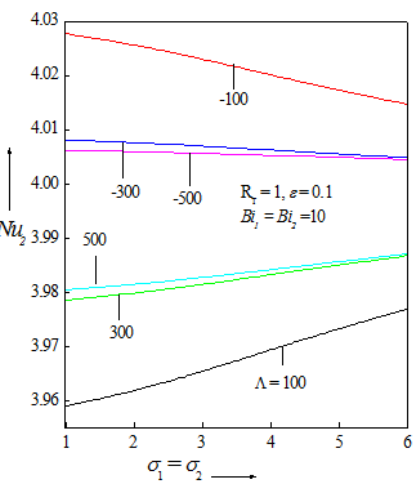

Fig.14b: Plots of $\mathrm{Nu}_{2}$ versus $\sigma_{1}=\sigma_{2}$ for different values of $\Lambda$
Tables 2-4 are the velocity and temperature solutions obtained by PM and DTM for symmetric and asymmetric wall heating conditions varying the perturbation parameter $\varepsilon$ for equal and unequal Biot numbers. In Table 2 , it is seen that in the absence of perturbation parameter, the PM and DTM solutions are equal for both the velocity and temperature fields. When the perturbation parameter $\varepsilon$ is 
increased $(\varepsilon=2)$, it is seen that the PM and DTM solutions do not agree. Similar nature is also observed in Table 3 and 4 for PM and DTM solutions. Table 2 and 3 are the solutions of velocity and temperature for asymmetric wall heating conditions for equal and unequal Biot numbers respectively. Table 2 and 3 also reveals that the percentage of error is large at the interface for velocity when compared with the error at the boundaries. Further the percentage of error between PM and DTM is large for unequal Biot numbers when compared with equal Biot numbers. Table 4 displays the solutions of symmetric wall heating conditions for equal Biot numbers. The percentage of error is less for symmetric wall heating conditions for equal Biot numbers when compared with asymmetric wall heat conditions.

Table 2: Values of velocity and temperature for $\Lambda=500$ and $R_{T}=1, \sigma_{1}=\sigma_{2}=4$

\begin{tabular}{|c|c|c|c|c|c|c|}
\hline \multirow{3}{*}{$y$} & \multicolumn{3}{|c|}{$\varepsilon=0, B i_{1}=B i_{2}=10$} & \multicolumn{3}{|c|}{$\varepsilon=2, B i_{1}=B i_{2}=10$} \\
\hline & Analytical & DTM & $\%$ Error & Analytical & DTM & \% Error \\
\hline & \multicolumn{6}{|l|}{ Velocity } \\
\hline-0.250 & 0.000000 & 0.000000 & $0.00 \%$ & 0.000000 & 0.000000 & $0.00 \%$ \\
\hline-0.150 & 0.045040 & 0.045040 & $0.00 \%$ & 0.145290 & 0.130350 & $1.49 \%$ \\
\hline-0.050 & 0.696740 & 0.696740 & $0.00 \%$ & 0.856920 & 0.833180 & $2.37 \%$ \\
\hline 0.000 & 1.055840 & 1.055840 & $0.00 \%$ & 1.227040 & 1.201710 & $2.53 \%$ \\
\hline 0.050 & 1.336910 & 1.336910 & $0.00 \%$ & 1.504990 & 1.480140 & $2.49 \%$ \\
\hline 0.150 & 1.345480 & 1.345480 & $0.00 \%$ & 1.461910 & 1.444680 & $1.72 \%$ \\
\hline \multirow[t]{2}{*}{0.250} & 0.000000 & 0.000000 & $0.00 \%$ & 0.000000 & 0.000000 & $0.00 \%$ \\
\hline & \multicolumn{6}{|l|}{ Temperature } \\
\hline-0.250 & -0.357140 & -0.357140 & $0.00 \%$ & -0.351460 & -0.352300 & $0.08 \%$ \\
\hline-0.150 & -0.214290 & -0.214290 & $0.00 \%$ & -0.203020 & -0.204740 & $0.17 \%$ \\
\hline-0.050 & -0.071430 & -0.071430 & $0.00 \%$ & -0.055800 & -0.058110 & $0.23 \%$ \\
\hline 0.000 & 0.000000 & 0.000000 & $0.00 \%$ & 0.016810 & 0.014350 & $0.25 \%$ \\
\hline 0.050 & 0.071430 & 0.071430 & $0.00 \%$ & 0.088740 & 0.086200 & $0.25 \%$ \\
\hline 0.150 & 0.214290 & 0.214290 & $0.00 \%$ & 0.231080 & 0.228580 & $0.25 \%$ \\
\hline 0.250 & 0.357140 & 0.357140 & $0.00 \%$ & 0.368970 & 0.367260 & $0.17 \%$ \\
\hline
\end{tabular}

Table 3: Values of velocity and temperature for $\Lambda=500$ and $R_{T}=1, \sigma_{1}=\sigma_{2}=4$

\begin{tabular}{lllllll}
\hline & \multicolumn{2}{l}{$\varepsilon=0, B i_{1}=1, B i_{2}=10$} & \multicolumn{2}{l}{$\varepsilon=2, B i_{1}=1, B i_{2}=10$} \\
\cline { 2 - 7 } & Analytical & DTM & \% Error & Analytical & DTM & \% Error \\
\cline { 2 - 7 } & Velocity & & & & & $0.00 \%$ \\
-0.250 & 0.000000 & 0.000000 & $0.00 \%$ & 0.000000 & 0.000000 & $3.72 \%$ \\
-0.150 & 0.410790 & 0.410790 & $0.00 \%$ & 0.548700 & 0.511480 & $5.27 \%$ \\
-0.050 & 0.876790 & 0.876790 & $0.00 \%$ & 1.074650 & 1.021960 & $5.35 \%$ \\
0.000 & 1.055840 & 1.055840 & $0.00 \%$ & 1.257930 & 1.204450 & $5.01 \%$ \\
0.050 & 1.156860 & 1.156860 & $0.00 \%$ & 1.347420 & 1.297300 & $3.20 \%$ \\
0.150 & 0.979730 & 0.979730 & $0.00 \%$ & 1.102830 & 1.070860 & $0.00 \%$ \\
0.250 & 0.000000 & 0.000000 & $0.00 \%$ & 0.000000 & 0.000000 & \\
& Temperature & & & & -0.142890 & $0.53 \%$ \\
-0.250 & -0.156250 & -0.156250 & $0.00 \%$ & -0.137610 & -0.079350 & $0.55 \%$ \\
-0.150 & -0.093750 & -0.093750 & $0.00 \%$ & -0.073830 & -0.016800 & $0.53 \%$ \\
-0.050 & -0.031250 & -0.031250 & $0.00 \%$ & -0.011520 & 0.014040 & $0.50 \%$ \\
0.000 & 0.000000 & 0.000000 & $0.00 \%$ & 0.019070 & 0.044620 & $0.47 \%$ \\
0.050 & 0.031250 & 0.031250 & $0.00 \%$ & 0.049320 & 0.105070 & $0.38 \%$ \\
0.150 & 0.093750 & 0.093750 & $0.00 \%$ & 0.108880 & 0.163330 & $0.22 \%$ \\
0.250 & 0.156250 & 0.156250 & $0.00 \%$ & 0.165570 & \\
\hline
\end{tabular}


Table 4: Values of velocity and temperature for $R_{T}=0, \sigma_{1}=\sigma_{2}=4$

\begin{tabular}{|c|c|c|c|c|c|c|}
\hline \multirow{3}{*}{$y$} & \multicolumn{3}{|c|}{$\varepsilon=0, B i_{1}=B i_{2}=10$} & \multicolumn{3}{|c|}{$\varepsilon=2, B i_{1}=B i_{2}=10$} \\
\hline & Analytical & DTM & \% Error & Analytical & DTM & $\%$ Error \\
\hline & \multicolumn{6}{|l|}{ Velocity } \\
\hline-0.250 & 0.000000 & 0.000000 & $0.00 \%$ & 0.000000 & 0.000000 & $0.00 \%$ \\
\hline-0.150 & 0.695260 & 0.695260 & $0.00 \%$ & 0.744640 & 0.738170 & $0.65 \%$ \\
\hline-0.050 & 1.016820 & 1.016820 & $0.00 \%$ & 1.091330 & 1.081560 & $0.98 \%$ \\
\hline 0.000 & 1.055840 & 1.055840 & $0.00 \%$ & 1.133490 & 1.123300 & $1.02 \%$ \\
\hline 0.050 & 1.016820 & 1.016820 & $0.00 \%$ & 1.091330 & 1.081560 & $0.98 \%$ \\
\hline 0.150 & 0.695260 & 0.695260 & $0.00 \%$ & 0.744640 & 0.738170 & $0.65 \%$ \\
\hline \multirow[t]{2}{*}{0.250} & 0.000000 & 0.000000 & $0.00 \%$ & 0.000000 & 0.000000 & $0.00 \%$ \\
\hline & \multicolumn{6}{|l|}{ Temperature } \\
\hline-0.250 & 0.000000 & 0.000000 & $0.00 \%$ & 0.003950 & 0.003430 & $0.05 \%$ \\
\hline-0.150 & 0.000000 & 0.000000 & $0.00 \%$ & 0.006490 & 0.005640 & $0.09 \%$ \\
\hline-0.050 & 0.000000 & 0.000000 & $0.00 \%$ & 0.007420 & 0.006440 & $0.10 \%$ \\
\hline 0.000 & 0.000000 & 0.000000 & $0.00 \%$ & 0.007520 & 0.006530 & $0.10 \%$ \\
\hline 0.050 & 0.000000 & 0.000000 & $0.00 \%$ & 0.007420 & 0.006440 & $0.10 \%$ \\
\hline 0.150 & 0.000000 & 0.000000 & $0.00 \%$ & 0.006490 & 0.005640 & $0.09 \%$ \\
\hline 0.250 & 0.000000 & 0.000000 & $0.00 \%$ & 0.003950 & 0.003430 & $0.05 \%$ \\
\hline
\end{tabular}

\section{Conclusions}

The analytical (PM) and semi-analytical (DTM) solutions were found for the problem of steady laminar mixed convective flow in a vertical channel filled with composite porous medium using boundary conditions of third kind. It was concluded that the flow at each position was an increasing function of $\varepsilon$ for upward flow and decreasing function of $\varepsilon$ for downward flow. The porous parameter suppresses the flow for symmetric and asymmetric wall heating conditions for all the governing parameters. Flow reversal was observed for asymmetric wall heating for equal Biot numbers and there is no flow reversal for unequal Biot numbers. The viscosity ratio increases the flow in region-I and decreases in region-II for equal Biot numbers. The width ratio and conductivity ratio suppress the flow in both the regions for equal Biot numbers. Similar result was also observed by Prathap Kumar et al. [17] for isothermal boundary conditions. The Nusselt number at the cold wall was increasing function of $|\varepsilon|$ and decreasing function of $\sigma$. The Nusselt number at the hot wall was decreasing function of $|\varepsilon|$ and increasing function of $\sigma$. The percentage of error between PM and DTM agree very well for small values of perturbation parameter. Fixing equal values for viscosity, width and conductivity for fluids in both the regions we get back the results of Umavathi and Santosh [38] for one fluid model.

\section{Acknowledgments}

One of the authors JPK greatly acknowledge the funding support from the Major Research project supported by
University Grants Commission India (Grant No.41774/2012 (SR)).

\section{Nomenclature}

$A-$ Constant used in Eq.(18)

$B i_{1}, B i_{2}-$ Biot numbers

$B r-$ Brinkman number $\left(\mu_{1} U_{0}^{(1)^{2}} / k_{1} \Delta T\right)$

$c_{p}$ - Specific heat at constant pressure

$D a_{1}, D a_{2}-$ Darcy numbers in region-I and II

$g-$ Acceleration due to gravity

$G r-$ Grashof number $\left(g \beta_{1} D_{1}^{3} \Delta T / v_{1}^{2}\right)$

$k$ - Ratio of thermal conductivities $\left(k_{1} / k_{2}\right)$

$h-$ Width ratio $\left(h_{2} / h_{1}\right)$

$N u_{1}, N u_{2}-$ Nusselt numbers

$p$ - Non-dimensional pressure gradient

$P$ - Difference between pressure and hydrostatic pressure

$q_{1}, q_{2}$-External heat transfer coefficients

Re - Reynolds number $\left(D_{1} U_{0}^{(1)} / v_{1}\right)$

$R_{T}$ - Temperature difference ratio

$T$ - Temperature

$T_{q_{1}}, T_{q_{2}}$ - Reference temperatures of the external fluid

$T_{0}$-Reference temperature

$U_{1}, U_{2}$ - Velocity component in the $X$-direction in region-I and II 
$U_{0}^{(i)}$-Reference velocity

$u_{1}, u_{2}$ - Dimensionless velocity in the $X$-direction in

region-I and II

$X$-Stream wise coordinate

$x$-Dimensionless stream wise coordinate

$Y$-Transverse coordinate

$y$-Dimensionless transverse coordinate

\section{Greek Symbols}

$\alpha_{1}, \alpha_{2}-$ Thermal diffusivity in region-I and II

$\beta_{1}, \beta_{2}$-Thermal expansion coefficient in region-I and II

$\kappa_{1}, \kappa_{2}$-Permeability of porous medium in region-I and II

$\Delta T-$ Reference temperature difference $\left(T_{q_{2}}-T_{q_{1}}\right)$

$\varepsilon-$ Dimensionless parameter

$\theta_{1}, \theta_{2}$ - Dimensionaless temperatures in region-I and II

$\mu_{1}, \mu_{2}-$ Viscosity in region-I and II

$v_{1}, v_{2}-$ Kinematics viscosities in region-I and II

$\rho_{1}, \rho_{2}-$ Density of fluids in region-I and II

$\Lambda$-Dimensionless parameter $(\mathrm{Gr} / \mathrm{Re})$

\section{Subscripts}

1 and 2 reference quantities for Region-I and II, respectively

\section{References}

[1] G. Beavers, and D.D. Joseph, "Boundary conditions at a naturally permeable wall," J. Fluid Mech, vol. 30: pp. 197207, 1967.

[2] G. Neale, and W. Nadar, "Practical significance of Brinkman's extension of Darcy's law: Coupled parallel flows within a channel and a bounding porous medium," Can. J. Chem. Engrg, vol. 52, pp. 475- 478, 1974.

[3] K. Vafai, and S.J. Kim, "Fluid mechanics of the inter-phase region between a porous medium and a fluid layer-an exact solution,” Int. J. Heat Fluid Flow, vol. 11, pp.254-256, 1990.

[4] K. Vafai, and R. Thiyagaraja, "Analysis of flow and heat transfer at the interface region of a porous medium," Int. J. Heat Fluid Flow, vol. 30, pp. 1391-1405, 1987.

[5] K. Vafai, and S.J. Kim, "Analysis of surface enhancement by a porous substrate," J. Heat Transfer, vol. 112, pp. 700$706,1990$.

[6] S.J. Kim, and C.Y. Choi, "Convection heat transfer in porous and overlaying layers heated from below," Int. J. Heat Mass Transfer, vol. 39, pp. 319-329, 1996.

[7] D. Poulikakos, and M. Kazmierczak, "Forced convection in a duct partially filled with a porous material," J. Heat Transfer, vol. 109, pp.653-662, 1987.

[8] J.A. Ochoa-Tapie, and S. Whitaker, "Heat transfer at the boundary between a porous medium and a homogeneous fluid,” Int. J. Heat Mass Transfer, vol. 40, pp. 2691-2707, 1997.

[9] J.W. Paek, B.H. Kang, S.Y. Kim, and J.M. Hyan, "Effective thermal conductivity and permeability of aluminum foam materials,” Int. J. Thermophys, vol. 21, pp. 453-464, 2000.

[10] A.V. Kuznetsov, "Analytical study of fluid flow and heat transfer during forced convection in a composite channel partly filled with a Brinkman-Forchheimer porous medium," Flow Turbulence and Combustion, vol. 60, pp. 173-192, 1998.

[11] D.A. Nield, and A.V. Kuznetsov, "The effect of heterogeneity in forced convection in a porous medium: parallel plate channel or circular duct," Int. J. Heat Mass Transfer, vol. 43, pp. 4119-4134, 2000.

[12] M.S. Malashetty, J.C. Umavathi, and J. Prathap Kumar, "Convective Flow and Heat Transfer in an Inclined Composite Porous Medium,” J. Porous Media, vol. 4(1), pp. 15-22, 2001.

[13] M.S. Malashetty, J.C. Umavathi, and J. Prathap Kumar, "Two fluid flow and heat transfer in an inclined channel containing porous and fluid layer," Heat and Mass Transfer, vol. 40, pp. 871-876, 2004.

[14] J.C. Umavathi, Ali J Chamkha, Abdul Mateen, and AlMudhaf A, "Oscillatory flow and heat transfer in a horizontal composite porous medium channel," Int. J. Heat and Tech, vol. 25(2), pp. 75-86, 2006.

[15] J.C. Umavathi, M.S. Malashetty, and J. Prathap Kumar, "Flow and heat transfer in an inclined channel containing a porous layer sandwiched between two fluid layers," ASME, Modelling Measurement and Controlling, vol. 74, pp. 19-35, 2005.

[16] J.C. Umavathi, J. Prathap Kumar, and K.S.R. Sridhar, "Flow and heat transfer of poiseuille.couette flow in an inclined channel for composite porous medium," Int. J. Appl. Mech. Engg, vol. 15(1), pp. 249-266, 2010.

[17] J. Prathap Kumar, J.C. Umavathi, and Basavaraj M Biradar, "Mixed convection of composite potous medium in a vertical channel with asymmetrical wall heating conditions," J. Porous Media, vol. 13(3), pp. 271-285, 2010.

[18] J. Prathap Kumar, J.C. Umavathi, I. Pop, and Basavaraj M Biradar, "Fully developed mixed convection flow in a vertical channel containing porous and fluid layer with isothermal or isoflux boundaries," Trans. Porous Med, vol. 80, pp. 117-135, 2009.

[19] P. Wibulswas, "Laminar flow heat transfer in non circular ducts," Ph.D. Thesis, London University, 1966 (as reported by Shah and London in 1971)

[20] R.W. Lyczkowski, C.W. Solbrig, and D. Gidaspow, Forced convective heat transfer in rectangular ducts general case of wall resistance and peripheral conduction. 969. Institute of Gas Technology Tech. Info, Center File 3229, 3424S, State, Street, Chicago, Ill 60616 (as reported by Shah and London in 1971).

[21] V. Javeri, "Analysis of laminar thermal entrance region of elliptical and rectangular channels with Kantorowich method," Warme- und Stoffuberragung, vol. 9, pp. 85-98, 1976. 
[22] E. Hicken, and Das, Temperatur field in laminar durchstromten Kanalen mitechteckquerschnitt bei unterschiedlicher Beheizung der Kanalwade," Warme- und Stoffubertragung, vol. 1, pp. 98-104, 1968.

[23] E.M. Sparrow, R. Siegal, "Application of variational methods to the thermal entrance region of ducts," Int. J. Heat Mass Transfer, vol. 1, pp. 161-172, 1960.

[24] V. Javeri, Heat transfer in laminar entrance region of a flat channel for the temperature boundary condition of the third kind," Warne-und Stoffubertragung Thermo-and Fluid Dynamics, vol. 10, pp. 137-144, 1977.

[25] V. Javeri, "Laminar heat transfer in a rectangular channel for the temperature boundary conditions of third kind," Int. J, Heat Mass Transfer, vol. 10, pp. 1029-1034, 1978.

[26] E. Zanchini, Effect of viscous dissipation on mixed convection in a vertical channel with boundary conditions of the third kind," Int. J Heat and Mass Transfer, vol. 41, pp. $3949-3959,1998$.

[27] M. Kumari, and G. Nath, "Mixed convection boundary layer flow over a thin vertical cylinder with localized injection/suction and cooling/heating," Int. Heat mass Transfer, vol. 47, pp. 969-976, 2004.

[28] J.K. Zhou, "Differential transformation and its applications for electrical circuits," Huarjung University Press, 1986. (in Chinese)

[29] M.J. Jang, C.L. Chen, and Y.C. Liu, "Two-dimensional differential transform for partial differential equations," Appl. Math. Comput, vol 21, pp. 261-270, 2001.

[30] A. Kurnaz, and G. Oturanç, "The differential transform approximation for the system of ordinary differential equations," Int. J. Comput. Math, vol. 82, pp. 709-719, 2005 .
[31] A. Arikoglu, and I. Ozkol, "Solution of differentialdifference equations by using differential transform method," Appl. Math. Comput, vol. 181, pp. 153-62, 2006.

[32] A.S.V. Ravi Kanth, and K. Aruna, "Solution of singular twopoint boundary value problems using differential transformation method," Physics Letters A, vol. 372, pp. 4671-4673, 2008.

[33] M.J. Jang, Y.L. Yeh, C.L. Chen, and W.C. Yeh, "Differential transformation approach to thermal conductive problems with discontinuous boundary condition," Appl., Math. Comput, vol. 216, pp. 2339-2350, 2010.

[34] M.M. Rashidi, N. Laraqi, and S. M. Sadri, "A novel analytical solution of mixed convection about an inclined flat plate embedded in a porous medium using the DTMPadé,” Int. J. Thermal Sci, vol. 49, pp. 2405-2412, 2010.

[35] H. Yaghoobi, and M. Torabi, "The application of differential transformation method to nonlinear equations arising in heat transfer," Int. Commu. Heat and Mass Transfer, vol. 38, pp. 815-820, 2011

[36] M.M. Rashidi, O. Anwar Beg, and N. Rahimzadeh, "A generalized differential transform method for combined free and forced convection flow about inclined surfaces in porous media," Chem. Eng. Comm, vol. 199, pp. 257-282, 2012.

[37] A. Barletta, "Laminar mixed convection with viscous dissipation in a vertical channel," Int. J. Heat Mass Transfm, vol. 41, pp. 3501-3513, 1998

[38] J.C. Umavathi, and V. Santosh, "Non-Darcy mixed convection in a vertical porous channel with boundary conditions of third kind," Trans. Porous Media, vol. 95, pp. $111-131,2012$ 